\title{
Oscillation of Certain Emden-Fowler Dynamic Equations on Time Scales
}

\author{
Qiaoshun Yang, ${ }^{1}$ Lynn Erbe, ${ }^{2}$ and Baoguo Jia ${ }^{3}$ \\ ${ }^{1}$ Department of Mathematics and Computer Science, Normal College, Jishou University, Jishou, Hunan 416000, China \\ ${ }^{2}$ Department of Mathematics, University of Nebraska-Lincoln, Lincoln, NE 68588-0130, USA \\ ${ }^{3}$ School of Mathematics and Computer Science, Zhongshan University, Guangzhou 510275, China
}

Correspondence should be addressed to Baoguo Jia; mcsjbg@mail.sysu.edu.cn

Received 4 April 2014; Accepted 19 May 2014; Published 26 May 2014

Academic Editor: Douglas R. Anderson

Copyright (C) 2014 Qiaoshun Yang et al. This is an open access article distributed under the Creative Commons Attribution License, which permits unrestricted use, distribution, and reproduction in any medium, provided the original work is properly cited.

We deal with the oscillation of a generalized Emden-Fowler dynamic equation in the form $\left(r(t)\left|Z^{\Delta}(t)\right|^{\alpha-1} Z^{\Delta}(t)\right)^{\Delta}+f(t, x(\delta(t)))=$ 0 . We establish some new oscillation criteria for the equation, which improve some of the main results of (H. Liu and P. Liu, 2013). Some examples are given to illustrate the new results.

\section{Introduction}

The theory of time scales has attracted a great deal of attention since it was first introduced by Hilger [1] in order to unify continuous and discrete analysis. For completeness, we recall the following concepts related to the notion of time scales; see $[2,3]$ for more details. A time scale $\mathbb{T}$ is an arbitrary nonempty closed subset of the real numbers $\mathbb{R}$. In this paper, since we shall be concerned with the oscillatory behavior of solutions, we shall also assume that sup $\mathbb{T}=\infty$. We define the time scale interval $\left[t_{0}, \infty\right)_{\mathbb{T}}$ by $\left[t_{0}, \infty\right)_{\mathbb{T}}:=\left[t_{0}, \infty\right) \cap \mathbb{T}$. The forward and backward jump operators are defined by

$$
\begin{aligned}
& \sigma(t):=\inf \{s \in \mathbb{T}: s>t\}, \\
& \rho(t):=\sup \{s \in \mathbb{T}, s<t\},
\end{aligned}
$$

where $\inf \emptyset:=\sup \mathbb{\mathbb { V }}$ and $\sup \emptyset:=\inf \mathbb{\mathbb { T }}$; here $\emptyset$ denotes the empty set. A point $t \in \mathbb{T}$ and $t>$ inf $\mathbb{T}$ is said to be leftdense if $\rho(t)=t$, right-dense if $t<\sup \mathbb{T}$ and $\sigma(t)=t$, left-scattered if $\rho(t)<t$, and right-scattered if $\sigma(t)>t$. The graininess function $\mu$ for the time scale $\mathbb{T}$ is defined by $\mu(t):=\sigma(t)-t$, and for any function $f: \mathbb{T} \rightarrow \mathbb{R}$, the notation $f^{\sigma}(t)$ denotes $f(\sigma(t))$. A function $g: \mathbb{T} \rightarrow \mathbb{R}$ is said to be rd-continuous provided $g$ is continuous at right-dense points and at left-dense points in $\mathbb{T}$ and left-hand limits exist and are finite. The set of all such rd-continuous functions is denoted by $C_{\text {rd }}(\mathbb{T})$. We say that $x: \mathbb{T} \rightarrow \mathbb{R}$ is differentiable at $t \in \mathbb{T}$ provided

$$
x^{\Delta}(t):=\lim _{s \rightarrow t} \frac{x(t)-x(s)}{t-s}
$$

exists when $\sigma(t)=t$ (here by $s \rightarrow t$ it is understood that $s$ approaches $t$ in the time scale) and when $x$ is continuous at $t$ and $\sigma(t)>t$

$$
x^{\Delta}(t):=\frac{x(\sigma(t))-x(t)}{\mu(t)} .
$$

Note that if $\mathbb{T}=\mathbb{R}$, then the delta derivative is just the standard derivative and when $\mathbb{T}=\mathbb{Z}$ the delta derivative is just the forward difference operator. The set of functions $f: \mathbb{T} \rightarrow \mathbb{R}$ which are differentiable and whose derivative is rd-continuous is denoted by $C_{\mathrm{rd}}^{1}(\mathbb{T}, \mathbb{R})$.

In this paper, we consider the oscillatory behavior of the nontrivial solutions of the second-order Emden-Fowler dynamic equation of the form

$$
\begin{array}{r}
\left(r(t)\left|Z^{\Delta}(t)\right|^{\alpha-1} Z^{\Delta}(t)\right)^{\Delta}+f(t, x(\delta(t)))=0, \\
t \in\left[t_{0}, \infty\right)_{\mathbb{T}}
\end{array}
$$


on an arbitrary time scale $\mathbb{T}$, with sup $\mathbb{T}=\infty$, where $Z(t)=$ $x(t)+p(t) x(\tau(t))$, and $\alpha>0$ is a constant. Throughout this paper, we always assume that

(A1) $r \in C_{\mathrm{rd}}\left(\left[t_{0}, \infty\right)_{\mathbb{T}},(0, \infty)\right)$ with $\int_{t_{0}}^{\infty} r^{-1 / \alpha}(t) \Delta t=\infty$;

(A2) $p \in C_{\mathrm{rd}}\left(\left[t_{0}, \infty\right)_{\mathbb{T}}, \mathbb{R}\right)$ with $0 \leq p(t)<1$;

(A3) $\tau, \delta \in C_{\mathrm{rd}}\left(\left[t_{0}, \infty\right)_{\mathbb{T}}, \mathbb{T}\right), \tau(t) \leq t, \delta(t) \leq t$, and $\lim _{t \rightarrow \infty} \tau(t)=\lim _{t \rightarrow \infty} \delta(t)=\infty ;$

(A4) $f(t, u) \in C\left(\left[t_{0}, \infty\right)_{\mathbb{T}} \times \mathbb{R}, \mathbb{R}\right)$ is a continuous function such that $u f(t, u)>0$, for all $u \neq 0$ and there exists a positive right-dense continuous function $q(t)$ defined on $\left[t_{0}, \infty\right)_{\mathbb{T}}$ such that $|f(t, u)| \geq q(t)|u|^{\beta}$ for all $t \in$ $\left[t_{0}, \infty\right)_{\mathbb{W}}$ and for all $u \in \mathbb{R}$, where $\beta>0$ is a constant.

By a solution of (4), we mean a nontrivial real-valued function $x \in C_{\mathrm{rd}}^{1}\left(\left[T_{x}, \infty\right), \mathbb{R}\right), T_{x} \geq t_{0}$ which has the property that $r(t)\left(Z^{\Delta}(t)\right)^{\alpha} \in C_{\mathrm{rd}}^{1}\left(\left[T_{x}, \infty\right), \mathbb{R}\right)$ and satisfies (4) that holds on $\left[T_{x}, \infty\right)$. The solutions vanishing in some neighborhood of infinity will be excluded from our consideration. A solution $x(t)$ of (4) is said to be oscillatory if it is neither eventually positive nor eventually negative. Otherwise it is said to be nonoscillatory. The equation itself is said to be oscillatory if all its solutions are oscillatory.

Recently, there has been an increasing interest in studying the oscillation behavior of second-order dynamic equations on time scales; see for example [4-10] and the references contained therein. In [5], the authors presented some criteria for the oscillation and asymptotic behavior of (4) in the case where

$$
\alpha \geq \beta>0, \quad \delta^{\Delta}(t)>0 .
$$

Also, we note further that in the proof of [5], the authors used the chain rule in the form

$$
\begin{gathered}
(x(\delta(t)))^{\Delta}=x^{\Delta}(\delta(t)) \delta^{\Delta}(t), \\
\delta(\sigma(t))=\sigma(\delta(t)) .
\end{gathered}
$$

So the natural question which arises is can we find some new oscillation conditions for (4) which do not require (5) and (6) and, in addition, improve the main results in [5]?

The purpose of this paper is to give an affirmative answer to this question. That is, we shall establish some new criteria for the oscillation of (4) which improve the main results in [5]. We also demonstrate that our results cover certain cases which were not covered in [5]. Finally, we give two examples to illustrate the main results.

\section{Main Results}

For notational simplicity, define

$$
\begin{aligned}
& R(t):=\int_{t_{0}}^{t} r^{-1 / \alpha}(s) \Delta s ; \\
& \theta(t, u):=\left(\int_{u}^{t} r^{-1 / \alpha}(s) \Delta s\right)^{-1} \int_{u}^{\delta(t)} r^{-1 / \alpha}(s) \Delta s \\
& t>u \geq t_{0} .
\end{aligned}
$$

We begin with the following lemmas.

Lemma 1. Assume that (4) has a positive solution $x(t)$ on $\left[t_{0}, \infty\right)_{\mathbb{T}}$. Then for sufficiently large $T$, one has

$$
\begin{array}{r}
Z(t)>0, \quad Z^{\Delta}(t)>0, \\
\left(r(t)\left|Z^{\Delta}(t)\right|^{\alpha-1} Z^{\Delta}(t)\right)^{\Delta} \leq 0, \\
t \in[T, \infty)_{\mathbb{V}} .
\end{array}
$$

Proof. Assume that (4) has a nonoscillatory solution on $\left[t_{0}, \infty\right)_{\mathbb{T}}$. Without loss of generality, we assume that there exists a $T \in\left[t_{0}, \infty\right)_{\mathbb{T}}$ such that $x(t), x(\tau(t)), x(\delta(t))>0$ on $[T, \infty)_{\mathbb{T}}$. Then it follows that $Z(t) \geq x(t)>0$. From (4), we have

$$
\left(r(t)\left|Z^{\Delta}(t)\right|^{\alpha-1} Z^{\Delta}(t)\right)^{\Delta}=-q(t) x^{\beta}(\delta(t)) \leq 0 .
$$

Hence, $r(t)\left|Z^{\Delta}(t)\right|^{\alpha-1} Z^{\Delta}(t)$ is decreasing on $[T, \infty)_{\mathbb{T}}$. We now claim that $Z^{\Delta}(t)>0$ on $t \in[T, \infty)_{\mathbb{T}}$. If not, then there exists a $t_{1} \in[T, \infty)_{\mathbb{T}}$ such that $Z^{\Delta}\left(t_{1}\right)<0$. Therefore,

$$
\begin{aligned}
& r(t)\left|Z^{\Delta}(t)\right|^{\alpha-1} Z^{\Delta}(t) \\
& \quad \leq r\left(t_{1}\right)\left|Z^{\Delta}\left(t_{1}\right)\right|^{\alpha-1} Z^{\Delta}\left(t_{1}\right):=-c<0, \quad t \geq t_{1},
\end{aligned}
$$

that is,

$$
Z^{\Delta}(t) \leq-\left[\frac{c}{r(t)}\right]^{1 / \alpha}
$$

Integrating (11) from $t_{1}$ to $t$, we find from $(A 1)$ that

$$
Z(t) \leq Z\left(t_{1}\right)-c^{1 / \alpha} \int_{t_{1}}^{t} \frac{1}{r^{1 / \alpha}(s)} \Delta s \longrightarrow-\infty \quad \text { as } t \rightarrow \infty
$$

which implies that $Z(t)$ is eventually negative. This contradicts the fact that $Z(t)>0$ on $[T, \infty)_{\mathbb{T}}$. Thus, $Z^{\Delta}(t)>0$ on $[T, \infty)_{\mathbb{T}}$. This completes the proof.

Lemma 2. Assume that (4) has a positive solution $x(t)$ on $\left[t_{0}, \infty\right)_{\mathbb{T}}$. Then for sufficiently large $t_{1}$,

$$
\frac{Z(\delta(t))}{Z(t)} \geq \theta\left(t, t_{1}\right), \quad t \geq t_{1} \text {. }
$$

Proof. As in the proof of Lemma 1 , there is a $t_{1} \in\left[t_{0}, \infty\right)_{\mathbb{T}}$ so that

$$
\begin{gathered}
Z(t)>0, \quad Z^{\Delta}(t)>0, \\
\left(r(t)\left(Z^{\Delta}(t)\right)^{\alpha}\right)^{\Delta} \leq 0, \\
t \in\left[t_{1}, \infty\right)_{\mathbb{T}} .
\end{gathered}
$$


Since $r(t)\left(Z^{\Delta}(t)\right)^{\alpha}$ is decreasing on $\left[t_{1}, \infty\right)_{\mathbb{T}}$, we can choose $t_{2}>t_{1}$ so that $\delta(t) \geq t_{1}$, for $t \geq t_{2}$. Then

$$
\begin{aligned}
Z(t)-Z(\delta(t)) & =\int_{\delta(t)}^{t} \frac{1}{r^{1 / \alpha}(s)}\left[r(s)\left(Z^{\Delta}(s)\right)^{\alpha}\right]^{1 / \alpha} \Delta s \\
& \leq\left[r(\delta(t))\left(Z^{\Delta}(\delta(t))\right)^{\alpha}\right]^{1 / \alpha} \int_{\delta(t)}^{t} \frac{1}{r^{1 / \alpha}(s)} \Delta s,
\end{aligned}
$$

consequently,

$$
\frac{Z(t)}{Z(\delta(t))} \leq 1+\frac{\left[r(\delta(t))\left(Z^{\Delta}(\delta(t))\right)^{\alpha}\right]^{1 / \alpha}}{Z(\delta(t))} \int_{\delta(t)}^{t} \frac{1}{r^{1 / \alpha}(s)} \Delta s .
$$

Also, we have, for $t \geq t_{2}$

$$
\begin{aligned}
& Z(\delta(t))>Z(\delta(t))-Z\left(t_{1}\right) \\
& \quad=\int_{t_{1}}^{\delta(t)} \frac{1}{r^{1 / \alpha}(s)}\left[r(s)\left(Z^{\Delta}(s)\right)^{\alpha}\right]^{1 / \alpha} \Delta s \\
& \quad \geq\left[r(\delta(t))\left(Z^{\Delta}(\delta(t))\right)^{\alpha}\right]^{1 / \alpha} \int_{t_{1}}^{\delta(t)} \frac{1}{r^{1 / \alpha}(s)} \Delta s,
\end{aligned}
$$

hence,

$$
\frac{\left[r(\delta(t))\left(Z^{\Delta}(\delta(t))\right)^{\alpha}\right]^{1 / \alpha}}{Z(\delta(t))} \leq\left(\int_{t_{1}}^{\delta(t)} \frac{1}{r^{1 / \alpha}(s)} \Delta s\right)^{-1} .
$$

Therefore, by combining inequalities (16) and (18) we have

$$
\frac{Z(t)}{Z(\delta(t))} \leq\left(\int_{t_{1}}^{t} \frac{1}{r^{1 / \alpha}(s)} \Delta s\right)\left(\int_{t_{1}}^{\delta(t)} \frac{1}{r^{1 / \alpha}(s)} \Delta s\right)^{-1}
$$

from which we have

$$
\frac{Z(\delta(t))}{Z(t)} \geq \theta\left(t, t_{1}\right)
$$

This completes the proof.

Lemma 3 (see [11]). Let $\phi(u)=a u-b u^{(\lambda+1) / \lambda}$, where $a \geq 0$, $b>0, \lambda>0$, and $u \in[0, \infty)$. Then

$$
\phi(u) \leq \frac{\lambda^{\lambda}}{(\lambda+1)^{\lambda+1}} \frac{a^{\lambda+1}}{b^{\lambda}} .
$$

For the positive solution $x(t)$ of (4), it follows from $Z(t)$ and Lemma 1 that, for $t \geq T$,

$$
\begin{aligned}
x(t) & =Z(t)-p(t) x(\tau(t)) \geq Z(t)-p(t) Z(\tau(t)) \\
& \geq(1-p(t)) Z(t),
\end{aligned}
$$

which implies

$$
x^{\beta}(\delta(t)) \geq(1-p(\delta(t)))^{\beta} Z^{\beta}(\delta(t)) .
$$

Combining (23) (A4), (4) one obtains

$$
\begin{aligned}
\left(r(t)\left(Z^{\Delta}(t)\right)^{\alpha}\right)^{\Delta} & \leq-q(t)(1-p(\delta(t)))^{\beta} Z^{\beta}(\delta(t)) \\
& =-\bar{p}(t) Z^{\beta}(\delta(t)),
\end{aligned}
$$

where $\bar{p}(t):=q(t)(1-p(\delta(t)))^{\beta}$.

One may now state and prove the main results. In these, one shall consider the two cases $\alpha \geq \beta$ and $\alpha<\beta$.

Theorem 4. Let $\alpha \geq \beta$. Assume that there exist a positive $r d$ continuous differentiable function $\xi(t)$ and a constant $M>0$ such that, for some $T \geq t_{0}$,

$$
\begin{aligned}
& \limsup _{t \rightarrow \infty} \int_{t_{0}}^{t}\left(\xi(s) \bar{p}(s) \theta^{\beta}(s, T)\right. \\
& \left.-\frac{M \alpha^{\alpha} r(s)(R(\sigma(s)))^{\alpha-\beta}\left(\xi_{+}^{\Delta}(s)\right)^{\alpha+1}}{(\alpha+1)^{\alpha+1} \beta^{\alpha} \xi^{\alpha}(s)}\right) \Delta s \\
& =\infty,
\end{aligned}
$$

where $\xi_{+}^{\Delta}(s):=\max \left\{\xi^{\Delta}(s), 0\right\}$. Then (4) is oscillatory on $\left[t_{0}, \infty\right)_{\mathbb{T}}$.

Proof. Let $x(t)$ be a nonoscillatory solution $x(t)$ of (4) on $\left[t_{0}, \infty\right)_{\mathbb{T}}$. Without loss of generality, we assume that there exists a $T \in\left[t_{0}, \infty\right)_{\mathbb{T}}$ (sufficiently large) such that $x(t), x(\tau(t)), x(\delta(t))>0$ on $[T, \infty)_{\mathbb{T}}$, and $Z(t)$ satisfies the conclusions of Lemmas 1 and 2 on $[T, \infty)_{\mathbb{T}}$. Consider the Riccati substitution

$$
w(t)=\xi(t) \frac{r(t)\left(Z^{\Delta}(t)\right)^{\alpha}}{Z^{\beta}(t)}, \quad t \geq T .
$$

Then $w(t)>0$. By [2, Theorem 1.20], Lemma 2, and (24), we have

$$
\begin{aligned}
w^{\Delta}(t)= & \left(r(t)\left(Z^{\Delta}(t)\right)^{\alpha}\right)^{\Delta} \frac{\xi(t)}{Z^{\beta}(t)} \\
& +\left(r(t)\left(Z^{\Delta}(t)\right)^{\alpha}\right)^{\sigma}\left(\frac{\xi(t)}{Z^{\beta}(t)}\right)^{\Delta} \\
\leq & -\xi(t) \bar{p}(t)\left(\frac{Z(\delta(t))}{Z(t)}\right)^{\beta}+\left(r(t)\left(Z^{\Delta}(t)\right)^{\alpha}\right)^{\sigma} \\
& \times \frac{\xi^{\Delta}(t) Z^{\beta}(t)-\xi(t)\left(Z^{\beta}(t)\right)^{\Delta}}{Z^{\beta}(t)\left(Z^{\beta}(t)\right)^{\sigma}} \\
\leq & -\xi(t) \bar{p}(t) \theta^{\beta}(t, T)+\frac{\xi^{\Delta}(t)}{\xi(\sigma(t))} w^{\sigma}(t) \\
& -\frac{\xi(t)}{\xi(\sigma(t))} w^{\sigma}(t) \frac{\left(Z^{\beta}(t)\right)^{\Delta}}{Z^{\beta}(t)} .
\end{aligned}
$$


By the Pötzsche chain rule [2, Theorem 1.87],

$$
\begin{aligned}
& \left(Z^{\beta}(t)\right)^{\Delta} \\
& \quad=\beta\left\{\int_{0}^{1}[(1-h) Z(t)+h Z(\sigma(t))]^{\beta-1} \mathrm{~d} h\right\} Z^{\Delta}(t) \\
& \quad \geq \begin{cases}\beta(Z(t))^{\beta-1} Z^{\Delta}(t), & \beta>1, \\
\beta(Z(\sigma(t)))^{\beta-1} Z^{\Delta}(t), & 0<\beta \leq 1 .\end{cases}
\end{aligned}
$$

Thus,

$$
\frac{\left(Z^{\beta}(t)\right)^{\Delta}}{Z^{\beta}(t)} \geq \begin{cases}\beta \frac{Z^{\Delta}(t)}{Z(t)}, & \beta>1, \\ \beta \frac{(Z(\sigma(t)))^{\beta-1}}{Z^{\beta}(t)} Z^{\Delta}(t), & 0<\beta \leq 1 .\end{cases}
$$

Noting that $Z(t)$ is increasing on $[T, \infty)_{\mathbb{T}}$, we get $Z(t) \leq$ $Z(\sigma(t))$ for $t \in[T, \infty)_{\mathbb{T}}$. Thus,

$$
\frac{\left(Z^{\beta}(t)\right)^{\Delta}}{Z^{\beta}(t)} \geq \beta \frac{Z^{\Delta}(t)}{Z(\sigma(t))} .
$$

Substituting (30) into (27), we obtain

$$
\begin{aligned}
w^{\Delta}(t) \leq & -\xi(t) \bar{p}(t) \theta^{\beta}(t, T)+\frac{\xi^{\Delta}(t)}{\xi(\sigma(t))} w^{\sigma}(t) \\
& -\frac{\beta \xi(t)}{\xi(\sigma(t))} w^{\sigma}(t) \frac{Z^{\Delta}(t)}{Z(\sigma(t))}, \quad t \geq T .
\end{aligned}
$$

Noting that $r^{1 / \alpha}(t) Z^{\Delta}(t)$ is decreasing, we have $r^{1 / \alpha}(t) Z^{\Delta}(t) \geq\left(r^{1 / \alpha}(t) Z^{\Delta}(t)\right)^{\sigma}$. It follows from the definition of $w(t)$ that

$$
Z^{\Delta}(t) \geq \frac{1}{(r(t) \xi(\sigma(t)))^{1 / \alpha}} w^{1 / \alpha}(\sigma(t)) Z^{\beta / \alpha}(\sigma(t)) .
$$

Substituting (32) into (31), we obtain

$$
\begin{aligned}
w^{\Delta}(t) \leq & -\xi(t) \bar{p}(t) \theta^{\beta}(t, T)+\frac{\xi^{\Delta}(t)}{\xi(\sigma(t))} w^{\sigma}(t) \\
& -\frac{\beta \xi(t) w^{(\alpha+1) / \alpha}(\sigma(t))}{r^{1 / \alpha}(t) \xi^{(\alpha+1) / \alpha}(\sigma(t)) Z^{(\alpha-\beta) / \alpha}(\sigma(t))}, \quad t \geq T .
\end{aligned}
$$

Since $r^{1 / \alpha}(t) Z^{\Delta}(t)$ is decreasing, there exists a constant $M_{1}>$ 0 such that $r^{1 / \alpha}(t) Z^{\Delta}(t) \leq M_{1}$ for $t \geq T$, which implies

$$
Z^{\Delta}(t) \leq \frac{M_{1}}{r^{1 / \alpha}(t)}, \quad t \geq T .
$$

Integrating both sides of (34) from $T$ to $t$, we get

$$
\begin{aligned}
Z(t) & \leq Z(T)+M_{1}(R(t)-R(T)) \\
& =R(t)\left(M_{1}+\frac{Z(T)-M_{1} R(T)}{R(t)}\right) .
\end{aligned}
$$

Hence, there exists a $T_{1} \geq T$ such that $Z(t) \leq\left(M_{1}+1\right) R(t)$ for $t \geq T_{1}$. Then,

$$
\begin{aligned}
& Z^{(\alpha-\beta) / \alpha}(\sigma(t)) \leq\left(M_{1}+1\right)^{(\alpha-\beta) / \alpha}(R(\sigma(t)))^{(\alpha-\beta) / \alpha} \\
& =M_{2}(R(\sigma(t)))^{(\alpha-\beta) / \alpha}, \quad t \geq T_{1},
\end{aligned}
$$

where $M_{2}=\left(M_{1}+1\right)^{(\alpha-\beta) / \alpha}$. Substituting (36) into (33), we get

$$
w^{\Delta}(t) \leq-\xi(t) \bar{p}(t) \theta^{\beta}(t, T)+\frac{\xi^{\Delta}(t)}{\xi(\sigma(t))} w^{\sigma}(t)
$$

$$
\begin{aligned}
& -\frac{\beta \xi(t) w^{(\alpha+1) / \alpha}(\sigma(t))}{M_{2} r^{1 / \alpha}(t) \xi^{(\alpha+1) / \alpha}(\sigma(t))(R(\sigma(t)))^{(\alpha-\beta) / \alpha}} \\
= & -\xi(t) \bar{p}(t) \theta^{\beta}(t, T)+\frac{\xi^{\Delta}(t)}{\xi(\sigma(t))} w^{\sigma}(t) \\
& -\Psi(t) w^{(\alpha+1) / \alpha}(\sigma(t)) \\
\leq & -\xi(t) \bar{p}(t) \theta^{\beta}(t, T)+\frac{\xi_{+}^{\Delta}(t)}{\xi(\sigma(t))} w^{\sigma}(t) \\
& -\Psi(t) w^{(\alpha+1) / \alpha}(\sigma(t)), \quad t \geq T_{1},
\end{aligned}
$$

where

$$
\Psi(t):=\frac{\beta \xi(t)}{M_{2} r^{1 / \alpha}(t) \xi^{(\alpha+1) / \alpha}(\sigma(t))(R(\sigma(t)))^{(\alpha-\beta) / \alpha}} .
$$

Taking $a=\xi_{+}^{\Delta}(t) / \xi(\sigma(t)), b=\Psi(t)$, from Lemma 3 and (37), we obtain

$$
\begin{aligned}
w^{\Delta}(t) \leq & -\xi(t) \bar{p}(t) \theta^{\beta}(t, T) \\
& +\frac{\alpha^{\alpha}}{(\alpha+1)^{\alpha+1} \Psi^{\alpha}(t)}\left(\frac{\xi_{+}^{\Delta}(t)}{\xi(\sigma(t))}\right)^{\alpha+1} \\
= & -\xi(t) \bar{p}(t) \theta^{\beta}(t, T) \\
& +\frac{M \alpha^{\alpha} r(t)(R(\sigma(t)))^{\alpha-\beta}\left(\xi_{+}^{\Delta}(t)\right)^{\alpha+1}}{(\alpha+1)^{\alpha+1} \beta^{\alpha} \xi^{\alpha}(t)}, \quad t \geq T_{1},
\end{aligned}
$$

where $M=M_{2}^{\alpha}$. Integrating both sides of (39) from $T_{1}$ to $t$, we have

$$
\begin{aligned}
& \int_{T_{1}}^{t}\left(\xi(s) \bar{p}(s) \theta^{\beta}(s, T)\right. \\
& \left.\quad-\frac{M \alpha^{\alpha} r(s)(R(\sigma(s)))^{\alpha-\beta}\left(\xi_{+}^{\Delta}(s)\right)^{\alpha+1}}{(\alpha+1)^{\alpha+1} \beta^{\alpha} \xi^{\alpha}(s)}\right) \Delta s \\
& \leq w\left(T_{1}\right)-w(t)<w\left(T_{1}\right) .
\end{aligned}
$$

Taking lim sup of both sides of this last inequality as $t \rightarrow \infty$, we get a contradiction to (25). This completes the proof. 
Theorem 5. Let $\alpha<\beta$. Assume that there exist a positive $r d$ continuous differentiable function $\xi(t)$ and a constant $K>0$ such that, for some $T \geq t_{0}$,

$$
\begin{aligned}
& \limsup _{t \rightarrow \infty} \int_{t_{0}}^{t}\left(\xi(s) \bar{p}(s) \theta^{\beta}(s, T)-\frac{\alpha^{\alpha} r(s)\left(\xi_{+}^{\Delta}(s)\right)^{\alpha+1}}{K(\alpha+1)^{\alpha+1} \beta^{\alpha} \xi^{\alpha}(s)}\right) \Delta s \\
& \quad=\infty,
\end{aligned}
$$

where $\xi_{+}^{\Delta}(s)$ is defined as Theorem 4. Then (4) is oscillatory on $\left[t_{0}, \infty\right)_{\mathbb{T}}$.

Proof. Assume that $x(t)$ is a nonoscillatory solution of (4). Proceeding as in the proof of Theorem 4 we get that (33) holds, that is,

$$
\begin{aligned}
w^{\Delta}(t) \leq & -\xi(t) \bar{p}(t) \theta^{\beta}(t, T)+\frac{\xi^{\Delta}(t)}{\xi(\sigma(t))} w^{\sigma}(t) \\
& -\frac{\beta \xi(t) w^{(\alpha+1) / \alpha}(\sigma(t))}{r^{1 / \alpha}(t) \xi^{(\alpha+1) / \alpha}(\sigma(t))} Z^{(\beta-\alpha) / \alpha}(\sigma(t)), \quad t \geq T .
\end{aligned}
$$

Since $\beta>\alpha$ and $Z(t)$ is increasing on $[T, \infty)_{\mathbb{T}}$, then there exist a $T_{2} \geq T$ and a positive constant $c_{1}$ such that $Z^{(\beta-\alpha) / \alpha}(\sigma(t)) \geq$ $c_{1}$ for $t \geq T_{2}$. Consequently,

$$
\begin{aligned}
w^{\Delta}(t) \leq & -\xi(t) \bar{p}(t) \theta^{\beta}(t, T)+\frac{\xi^{\Delta}(t)}{\xi(\sigma(t))} w^{\sigma}(t) \\
& -\frac{c_{1} \beta \xi(t) w^{(\alpha+1) / \alpha}(\sigma(t))}{r^{1 / \alpha}(t) \xi^{(\alpha+1) / \alpha}(\sigma(t))}, \quad t \geq T_{2} .
\end{aligned}
$$

Let

$$
\bar{\Psi}(t):=\frac{c_{1} \beta \xi(t)}{r^{1 / \alpha}(t) \xi^{(\alpha+1) / \alpha}(\sigma(t))},
$$

then $\bar{\Psi}(t)>0$, and

$$
\begin{aligned}
w^{\Delta}(t) \leq & -\xi(t) \bar{p}(t) \theta^{\beta}(t, T)+\frac{\xi_{+}^{\Delta}(t)}{\xi(\sigma(t))} w(\sigma(t)) \\
& -\bar{\Psi}(t) w^{(\alpha+1) / \alpha}(\sigma(t)), \quad t \geq T_{2} .
\end{aligned}
$$

The remainder of the proof is similar to that of Theorem 4 and is therefore omitted. This completes the proof for the case $\alpha<\beta$.

Remark 6. Theorems 4 and 5 remove the Conditions (5) and (6). Moreover, the authors in [5] established oscillation theorems for (4) only for the case $\alpha \geq \beta>0$. Our results here hold without this assumption, so our results improve the main results [5].

Remark 7. The results established here are valid for general time scales, with no additional restrictions, for example, $\mathbb{T}=$ $\mathbb{R}, \mathbb{T}=\mathbb{Z}$, and $\mathbb{T}=h \mathbb{Z}$ with $h>0, \mathbb{T}=q^{\mathbb{N}_{0}}$ with $q>1$, and $\mathbb{T}=\mathbb{N}_{0}^{2}$; see $[2,3]$.

\section{Some Examples}

In this section, we give two examples to illustrate our main results.

Example 1. Let $\mathbb{T}=2^{\mathbb{N}_{0}}\left(\mathbb{N}_{0}=\mathbb{N} \bigcup\{0\}\right), \alpha=3, \beta=2$. Consider the neutral nonlinear dynamic equation

$$
\begin{array}{r}
\Delta_{2}\left(\Delta_{2} Z\left(2^{k}\right)\right)^{3}+\frac{\delta^{2}\left(2^{k}\right)}{\theta^{2}\left(2^{k}, 1\right)}\left|x\left(\delta\left(2^{k}\right)\right)\right| x\left(\delta\left(2^{k}\right)\right)=0, \\
k_{0}=0,
\end{array}
$$

where $\tau\left(2^{k}\right)$ satisfies $(A 3)$, and $Z\left(2^{k}\right)=x\left(2^{k}\right)+\left(2^{k}-\right.$ 1) $/ 2^{k} x\left(\tau\left(2^{k}\right)\right)$.

Here,

$$
r\left(2^{k}\right)=1, \quad p\left(2^{k}\right)=\frac{2^{k}-1}{2^{k}}, \quad q\left(2^{k}\right)=\frac{\delta^{2}\left(2^{k}\right)}{\theta^{2}\left(2^{k}, 1\right)}
$$

It is clear that $(A 1)$ holds, and $\bar{p}\left(2^{k}\right)=q\left(2^{k}\right)\left(1-p\left(\delta\left(2^{k}\right)\right)\right)^{\beta}=$ $1 / \theta^{2}\left(2^{k}, 1\right), R\left(\sigma\left(2^{k}\right)\right)=2^{k+1}-1$.

Let $\xi\left(2^{k}\right)=2^{k}$. Noting that $\sum_{k=0}^{\infty} r^{-1 / \alpha}\left(2^{k}\right) 2^{k}=\infty$ implies $\lim _{k \rightarrow \infty} \theta\left(2^{k}, 2^{k_{T}}\right) / \theta\left(2^{k}, 1\right)=1$ for $k_{T} \geq 1$, we get

$$
\begin{gathered}
\limsup _{t \rightarrow \infty} \int_{t_{0}}^{t}\left(\xi(s) \bar{p}(s) \theta^{\beta}(s, T)\right. \\
\left.-\frac{M \alpha^{\alpha} r(s)(R(\sigma(s)))^{\alpha-\beta}\left(\xi_{+}^{\Delta}(s)\right)^{\alpha+1}}{(\alpha+1)^{\alpha+1} \beta^{\alpha} \xi^{\alpha}(s)}\right) \Delta s \\
=\limsup _{k \rightarrow \infty} \sum_{i=0}^{k-1}\left(2^{i} \frac{\theta^{2}\left(2^{i}, 2^{k_{T}}\right)}{\theta^{2}\left(2^{i}, 1\right)}-\frac{3^{3} M\left(2^{i+1}-1\right)}{4^{4} 2^{3} 2^{2 i}}\right) 2^{i} \\
\geq \limsup _{k \rightarrow \infty} \sum_{i=0}^{k-1}\left(2^{i}-\frac{M\left(2^{i+1}-1\right)}{2^{2 i}}\right) 2^{i}=\infty .
\end{gathered}
$$

Thus, by Theorem 4, (46) is oscillatory.

Example 2. Consider the neutral dynamic equation

$$
\begin{aligned}
\left(\frac{1}{\sigma^{1+\alpha}(t)}\left|Z^{\Delta}(t)\right|^{\alpha-1} Z^{\Delta}(t)\right)^{\Delta} & \\
+\frac{(1+\delta(t))^{\beta}}{\delta^{\beta}(t) \theta^{\beta}\left(t, t_{0}\right)}|x(\delta(t))|^{\beta-1} x(\delta(t)) & =0 \\
t_{0} & >0
\end{aligned}
$$

where $\beta>\alpha>0$ are constants, $\tau(t)$ satisfies $(A 3)$, and $Z(t)=$ $x(t)+1 /(t+1) x(\tau(t))$. 
For (4), we let

$$
r(t)=\frac{1}{\sigma^{1+\alpha}(t)}, \quad p(t)=\frac{1}{t+1}, \quad q(t)=\frac{(1+\delta(t))^{\beta}}{\delta^{\beta}(t) \theta^{\beta}\left(t, t_{0}\right)} .
$$

Since

$$
\int_{t_{0}}^{\infty} \frac{1}{r^{1 / \alpha}(s)} \Delta s=\int_{t_{0}}^{\infty} \sigma^{(1+\alpha) / \alpha}(s) \Delta s=\infty,
$$

then $(A 1)$ holds and $\bar{p}(t)=q(t)(1-p(\delta(t)))^{\beta}=1 / \theta^{2}\left(t, t_{0}\right)$.

Let $\xi(t)=t$. Noting that $\int_{t_{0}}^{\infty} r^{-1 / \alpha}(t) \Delta t=\infty$ implies $\lim _{t \rightarrow \infty} \theta(t, T) / \theta\left(t, t_{0}\right)=1$ for $T \geq t_{0}$, we have

$$
\begin{aligned}
& \limsup _{t \rightarrow \infty} \int_{t_{0}}^{t}\left(\xi(s) \bar{p}(s) \theta^{\beta}(s, T)\right. \\
& \left.\quad-\frac{\alpha^{\alpha} r(s)\left(\xi_{+}^{\Delta}(s)\right)^{\alpha+1}}{K(\alpha+1)^{\alpha+1} \beta^{\alpha} \xi^{\alpha}(s)}\right) \Delta s \\
& =\limsup _{t \rightarrow \infty} \int_{t_{0}}^{t}\left(s \frac{\theta^{2}(s, T)}{\theta^{2}\left(s, t_{0}\right)}-\frac{\alpha^{\alpha}}{K(\alpha+1)^{\alpha+1} \beta^{\alpha} \sigma^{1+\alpha}(s) s^{\alpha}}\right) \Delta s \\
& \geq \limsup _{t \rightarrow \infty} \int_{t_{0}}^{t}\left(s-\frac{1}{K \beta^{\alpha} s^{1+2 \alpha}}\right) \Delta s \\
& \geq \limsup _{t \rightarrow \infty} \frac{1}{2} \int_{t_{0}}^{t} s \Delta s=\infty .
\end{aligned}
$$

Thus, by Theorem 5, (49) is oscillatory.

\section{Conflict of Interests}

The authors declare that there is no conflict of interests regarding the publication of this paper.

\section{Acknowledgments}

Qiaoshun Yang was supported by The Foundation of Hunan Educational Department (no. 13C753) and The Main Foundation of Jishou University (no. 2012JSUJGA23). The work of Lynn Erbe was performed during a visit to Zhongshan (Sun Yat-sen) University in Guangzhou, China. Baoguo Jia was supported by The National Natural Science Foundation of China (no. 11271380); The Guangdong Province Key Laboratory of Computational Science; and The Guangdong Province Natural Science Foundation (S2013010013050).

\section{References}

[1] S. Hilger, "Analysis on measure chains-a unified approach to continuous and discrete calculus," Results in Mathematics, vol. 18, no. 1-2, pp. 18-56, 1990.

[2] M. Bohner and A. Peterson, Dynamic Equations on Time Scales: An Introduction with Applications, Birkhäuser, Boston, Mass, USA, 2001.
[3] M. Bohner and A. Peterson, Advances in Dynamic Equations on Time Scales, Birkhäuser, Boston, Mass, USA, 2003.

[4] A. Douglas and S. H. Saker, "Interval oscillation criteria for forced Emden-Fowler functional dynamic equations with oscillatory potential," Science China Mathematics, vol. 56, no. 3, pp. 561-576, 2013.

[5] H. Liu and P. Liu, "Oscillation criteria for some new generalized Emden-Fowler dynamic equations on time scales," Abstract and Applied Analysis, vol. 2013, Article ID 962590, 16 pages, 2013.

[6] D.-X. Chen, "Oscillation of second-order Emden-Fowler neutral delay dynamic equations on time scales," Mathematical and Computer Modelling, vol. 51, no. 9-10, pp. 1221-1229, 2010.

[7] L. Erbe, B. Jia, and A. Peterson, "On the asymptotic behavior of solutions of Emden-Fowler equations on time scales," Annali di Matematica Pura ed Applicata, vol. 191, no. 2, pp. 205-217, 2012.

[8] Q. Yang and Z. Xu, "Oscillation criteria for second order quasilinear neutral delay differential equations on time scales," Computers \& Mathematics with Applications, vol. 62, no. 10, pp. 3682-3691, 2011.

[9] S. H. Saker and D. O'Regan, "New oscillation criteria for second-order neutral functional dynamic equations via the generalized Riccati substitution," Communications in Nonlinear Science and Numerical Simulation, vol. 16, no. 1, pp. 423-434, 2011.

[10] Z. Han, T. Li, S. Sun, and C. Zhang, "Oscillation behavior of third-order neutral Emden-Fowler delay dynamic equations on time-scales," Advances in Difference Equations, vol. 2010, Article ID 586312, 23 pages, 2010.

[11] G. H. Hardy, J. E. Littlewood, and G. Pólya, Inequalities, Cambridge University Press, Cambridge, UK, 2nd edition, 1952. 


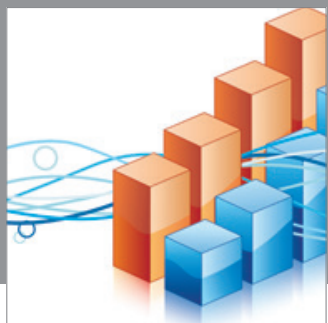

Advances in

Operations Research

mansans

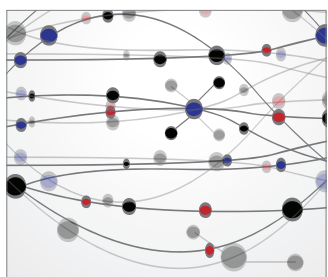

The Scientific World Journal
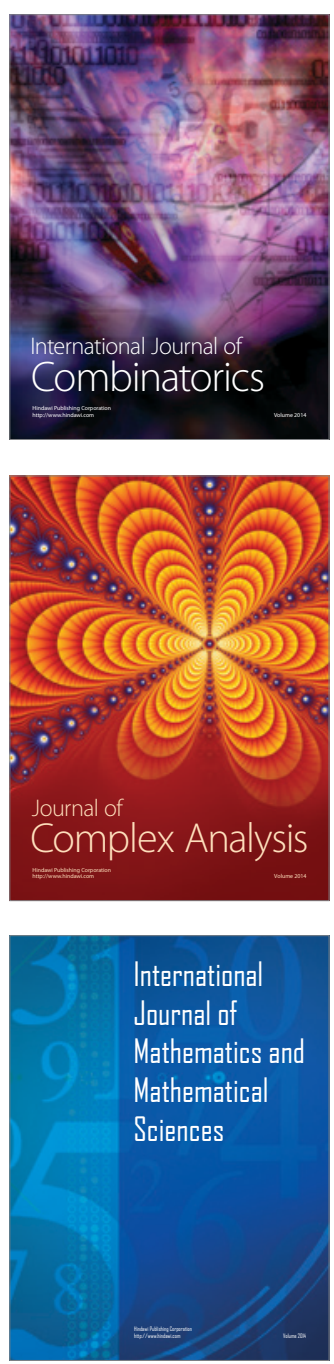
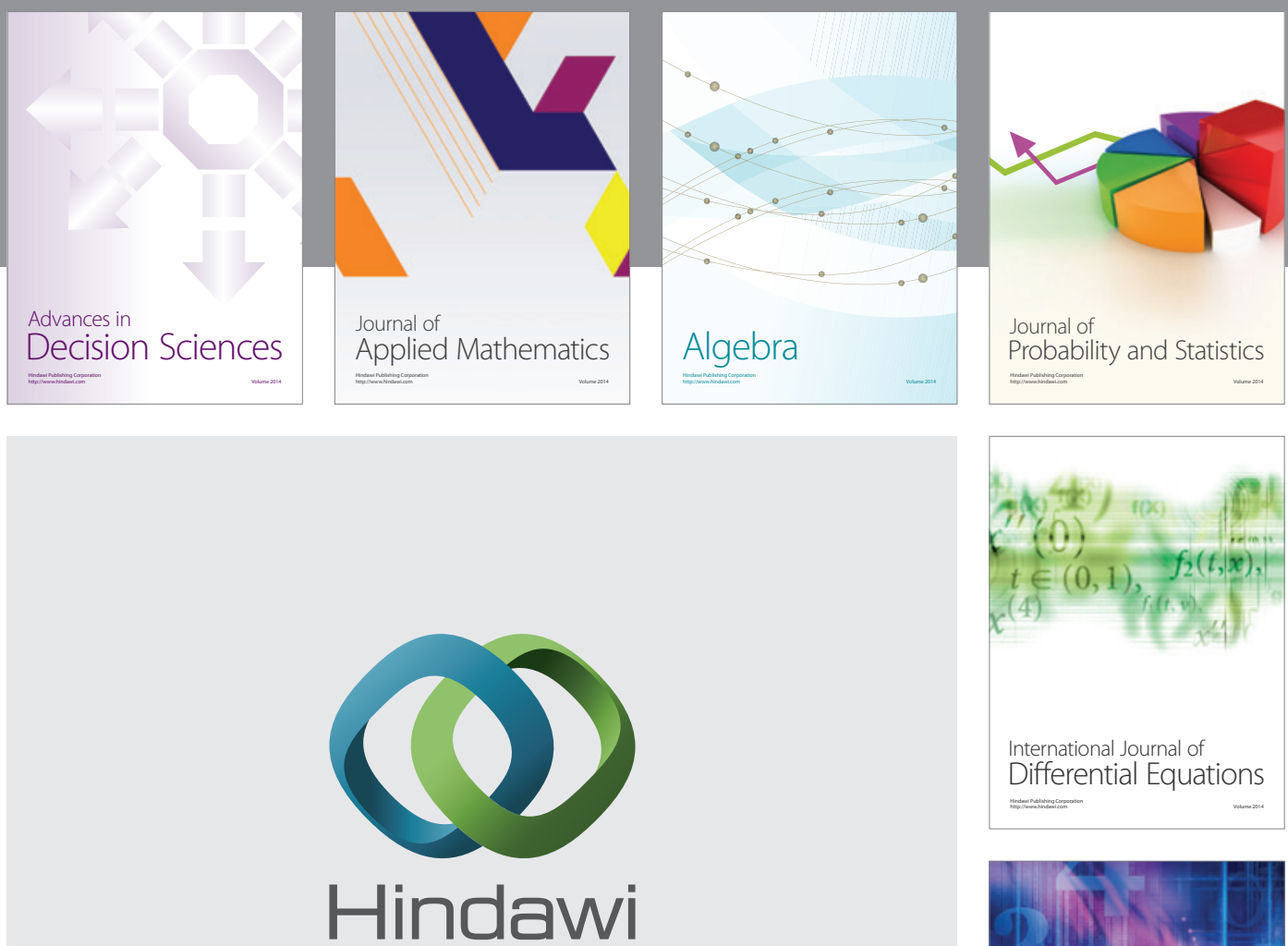

Submit your manuscripts at http://www.hindawi.com
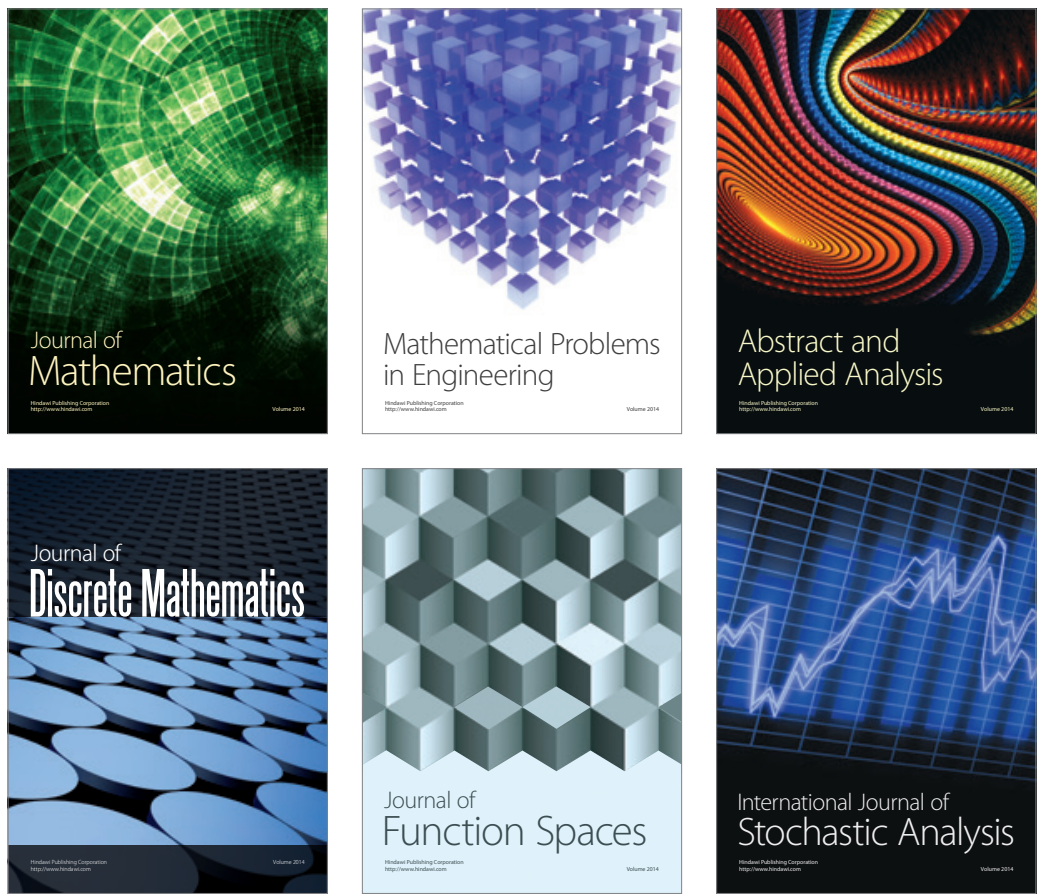

Journal of

Function Spaces

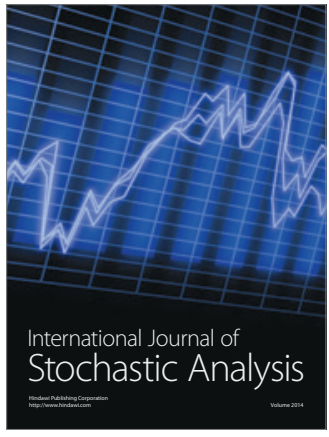

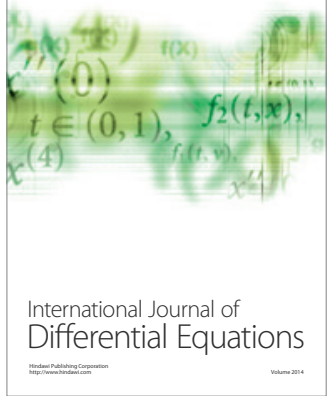
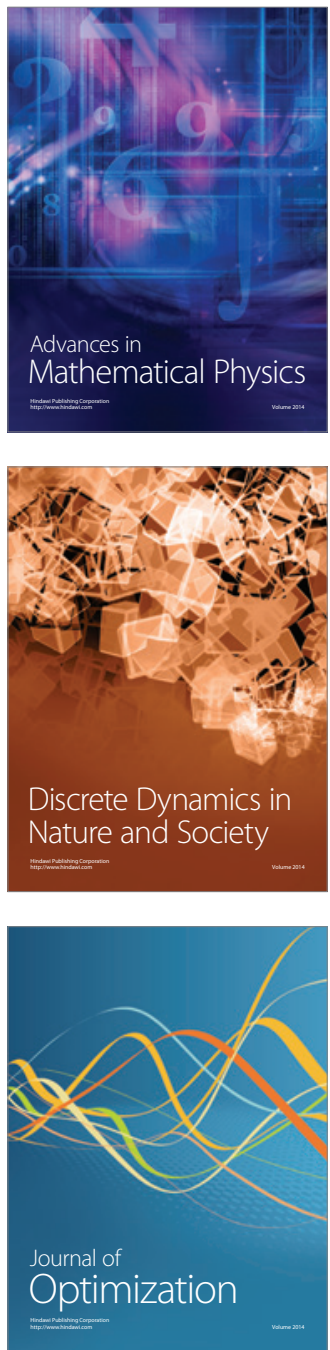